\title{
Research on Limit Analysis Finite Element Method for Loess Slope and Application
}

\author{
Jia Yajun ${ }^{1,2}$, Chen Cunli ${ }^{2}$, Hu Baocheng ${ }^{1}, \quad$ Zhang Huyuan $^{3}$, Ma li $^{1}$ \\ ${ }^{1}$ Gansu Forestry Technological College, Tianshui, Gansu 741020, China \\ ${ }^{2}$ Civil Engineering and Architecture Institute, Xi'an University of Technology, Xi'an 710048, China \\ ${ }^{3}$ Lanzhou University, Lanzhou, Gansu 730000, China
}

Keywords: Limit Analysis FEM, Geotechnical Engineering; Side Slope

\begin{abstract}
Classical geotechnical engineering limit element analysis method is widely used, but it is not suitable for heterogeneous materials. In particular, the finite element method should be used to analyze the strength of the loess slope. With the development of computer technology, it is possible to utilize computer technology to finite element analysis on the strength of loess slope. This paper introduce the limit analysis finite element method basic principle, including the definition of safety factor, rock and soil as a whole lost stability judgment method, the experimental data show that the method possessed good effect to loess landslide prediction.
\end{abstract}

\section{Theoretical Introduction of Limit Analysis FEM}

D Limit analysis is an important branch of geotechnical engineering stability analysis with slip line method and limit equilibrium analysis compared rigorous theoretical basis, while eliminating the need to solve the ultimate load as finite element or finite difference methods using incremental method while direct solution to the ultimate load.

Because finite element limit analysis method of the safety factor defined in terms of geotechnical engineering failure state occurs varies. As a class side (sliding) slope geotechnical engineering since the majority affected by the environment, geotechnical strength decreases, resulting in side (sliding) slope instability and failure. The introduction of the foundation or slope limit state, there are two ways: First, the incremental load, such as seeking the ultimate bearing capacity of the foundation; the second is strength reduction, such as safety factor of the slope ${ }^{[1]}$.

Currently, the standard soil destruction as the following: figurines of slipping through the region, indicating that the slip plane have reached the limit equilibrium state of each point. Finite element calculation does not converge to characterize the soil has been destroyed. Flag damage soil should be unlimited sliding movement of soil, then soil slip plane strain and displacement mutation and unlimited development. Application of FEM limit analysis conditions need to be met: there must be a mature and reliable finite element program; for practical constitutive model and yield strength criterion; the establishment of the calculation range, boundary conditions, meshing and other finite element model of the calculation parameters settings to meet the finite element calculation accuracy.

\section{Advantages of Limit Analysis FEM}

Numerical limit analysis method for solving process with traditional methods, calculated using strict ideal elastoplastic numerical method for the numerical calculation, by continuously reducing the material strength or load increases, so that in the final numerical calculation to destroy the state, this when the destruction of white face automatically generated and issued destroy information. Thus it does not presuppose failure surface, to destroy the strength reduction factor is stable when the safety factor, load is reached ultimate load at failure ${ }^{[2]}$. Fig.1 shows the advantages of limit analysis FEM. 


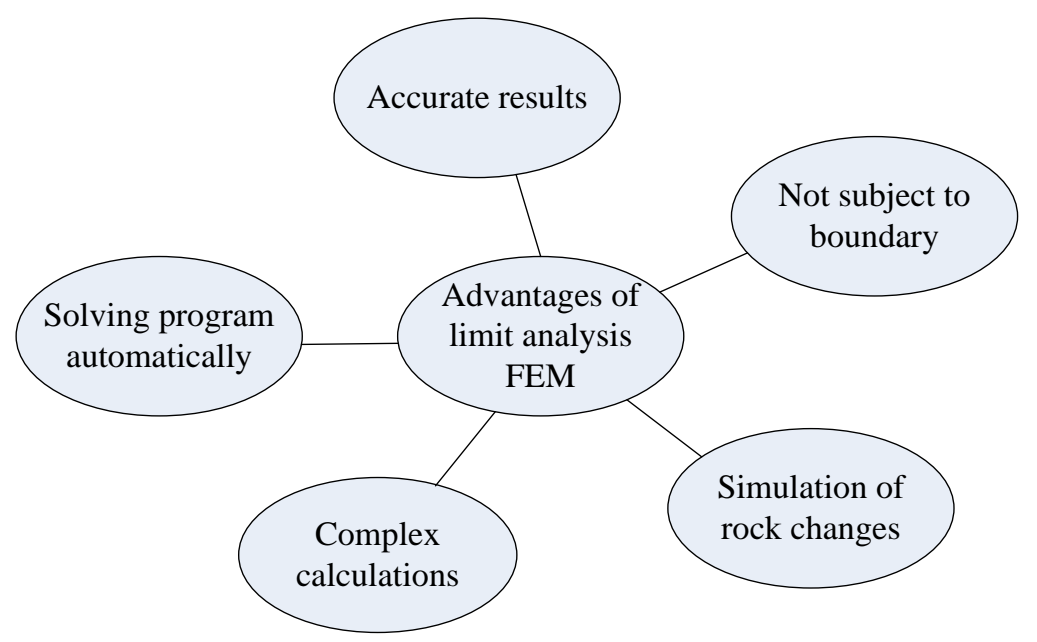

Fig. 1.The advantages of limit analysis FEM

The limit analysis FEM has both numerical methods and the advantages of the classical limit analysis method, numerical method has both advantages and wide adaptability, but also has the advantage of close limit analysis of loess slope design, practical. When using finite element strength reduction solving safety factor, without assuming that the shape and position of the sliding surface, but also without the slice, but is automatically determined by the program slip surface and strength reserve safety factor. Solving the ultimate bearing capacity finite element method overload, do not assume the position of failure surface and gives answers to the theory, and the program automatically given by the destruction mechanism and ultimate bearing capacity.

Numerical analysis method has various advantages, it is possible for complex topography, and geologic conditions would limit the variety of geotechnical engineering calculation, geometry from engineering, boundary conditions and material unevenness and the like. Consider stress-strain relationship can provide stress, strain, displacement and plastic zone and other forces and deformation of all the information. To consider the interaction of rock and soil and supporting structure, the simulated excavation process and progressive failure. The limit analysis method can use the power of internationally accepted procedures, the calculated results are accurate, clearly expressed, practical, convenient, which will lead to a major reform of geotechnical design methods, so it is a quite promising computing method ${ }^{[3]}$.

\section{Application in Slope Engineering}

Currently, the limit of finite element analysis method is mainly used side (sliding) slope stability calculation and control engineering calculations. Anti-dumping layered rock slope, when the inclination is large rock, often prone to toppling. Because of the larger rock inclination, to produce the anti-dumping layered rock slope outside the bending deformation, cracking and breaking destruction, dump side slope instability.

Slope stabilization wedge is a typical three-dimensional limit equilibrium problem, undermining wedge combination of two sets of structural planes and free face together. Were investigated geometry symmetrical and asymmetrical wedge two cases, its geometry, physical parameters, the material parameter. Finite element modeling, simulation press have weak layer thickness of the surface structure. Press spatial model calculations yield criterion using M-C guidelines or D-P guidelines Mohr-Coulomb equivalent area circle DP3 guidelines, safety factor finite element strength reduction method. When there are multiple sets of dumping to the slope surface structure, especially in the presence of approximately vertical surface structure, easy to block rock-fall occurred. A set of main vertical structural plane, another group of obliquity of 200, calculated using the discrete element method, there has been mass collapse destroyed ${ }^{[4]}$. Fig.2 shows the failure characteristics of tilting loess slope. 

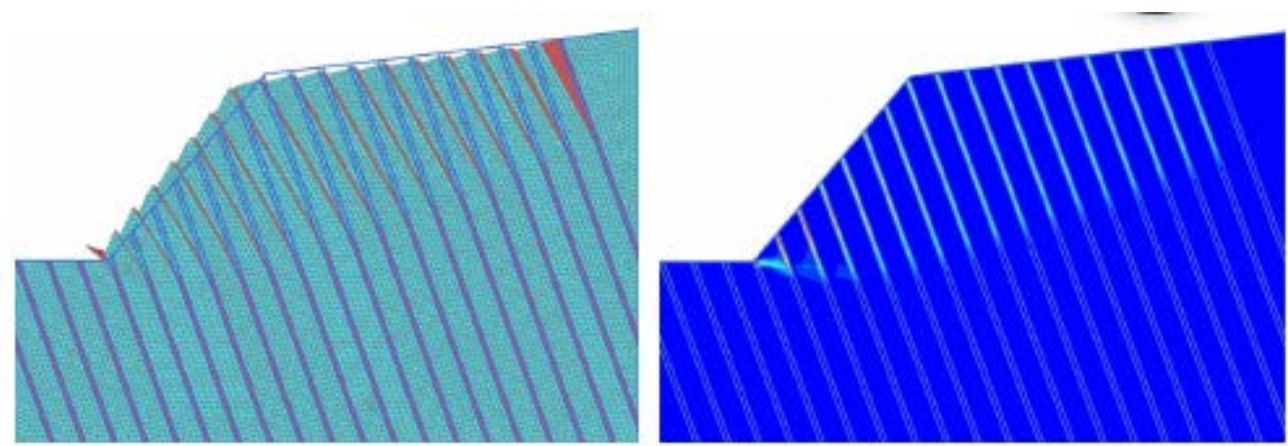

Fig. 2 .The failure characteristics of tilting loess slope

Side slope occurs mainly along the three-dimensional structure of the wedge surface ${ }^{[5]}$, the performance of the sliding broken field. Symmetric wedge model calculation as follows: the equivalent plastic strain diagram. Press the safety factor finite element strength reduction obtained was 1.283, the safety factor calculated according to the conventional method was 1.293 , and the calculation error is $1 \%$. Fig.3 shows the equivalent plastic strain of asymmetry wedge body.

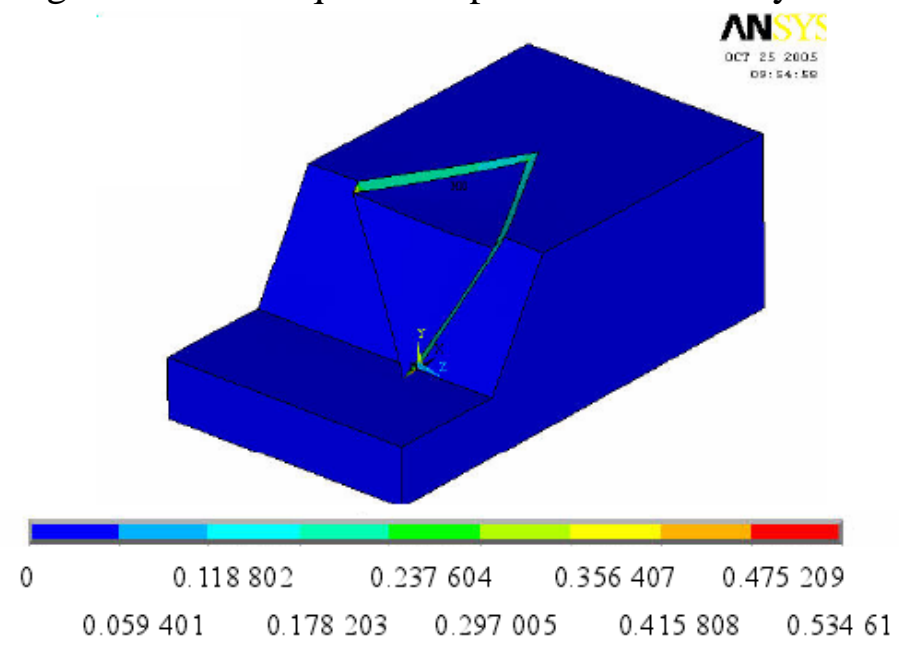

Fig. 3 .The control panel of system

\section{Improve the Calculation Accuracy}

In order to achieve accuracy, the general must meet the following conditions: to have a mature and reliable, powerful finite element program, especially the choice of internationally recognized generic programs that safe and reliable function and versatility. There are practical for geotechnical constitutive model and the strength criterion ${ }^{[6]}$.

For example, calculation range, boundary conditions, meshing, etc. can improve the finite element calculation accuracy. This experienced personnel calculation is not difficult, but some lack the computing experience that computing staff often due to improper handling resulting in insufficient accuracy. By the side slope numerical example presented below to the range of meshing process.

Boundary values in FEM calculation results have a greater opening to the Movies. When the slope angle of the distance to the left boundary of 1.5 times higher slope, the top of the hill from the right border of the slope to 25 times higher, and the upper and lower boundaries of not less than 2 times the total height of the slope is high, accuracy is desirable. Must be considered appropriate mesh density, if meshing process, can also be an important part of the calculation of partial encryption, unimportant place, you can sparse some should be noted that from dense to sparse best to have a smooth transition, not sudden and dramatic change in cell size. Fig.4 shows the FEM model with local grid refinement. 


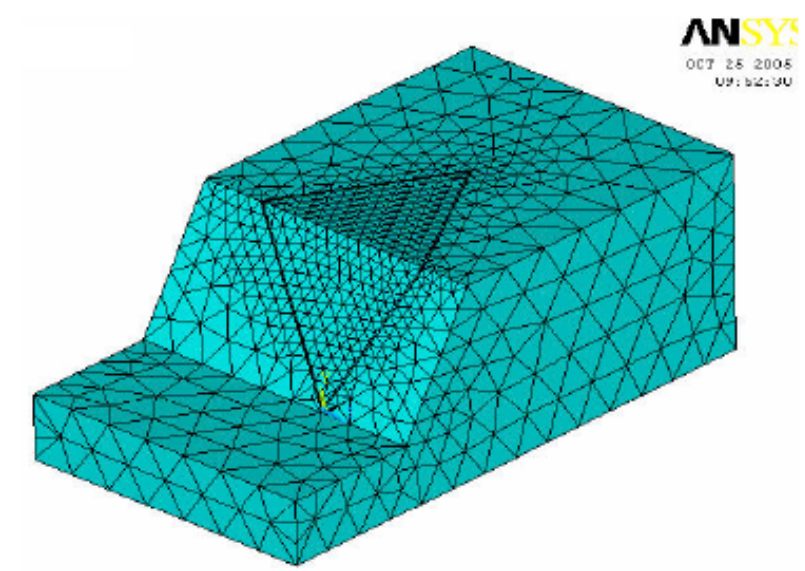

Fig. 4 .The FEM model with local grid refinement

\section{Conclusions}

The limit analysis FEM combines both numerical analysis and classical limit analysis method, and is particularly suitable for the analysis and design of geotechnical engineering. With the development of computer technology, geotechnical engineering finite element analysis method limit analysis is becoming a new branch. Because it may be the limiting analysis using finite element numerical analysis method operation, and have ready a large program available, thus expanding the scope of application of the limit analysis method. This method can be penetrating and non-penetrating jointed rock slope stability analysis, and can consider the impact of groundwater, the construction process can be considered interaction of various retaining structures and geotechnical material, which has a lot of advantages and application prospects..

\section{Acknowledgements}

The science and technology support program Foundation of Tianshui (No. 2015-25); Scientific research project of college in Gansu (2015A-188);

Gansu Education Science Planning Office (GS[2011] GHBG05、GS[2013] GHBZ092);Gansu forestry college teachers' Scientific Research Fund Project (GSLY-008)

\section{References}

[1] ZHANG Liming, ZHENG Yingren, WANG Zaiquan, et al. Application of strength reduction (mite element method to road tunnels [J]. Rock and Soil Mechanics, 2007, 28(1): 97-101.

[2] MUNOZ J J, BONET, et al. Upper and lower bounds in limit analysis: Adaptive meshing strategies and discontinuous loading [J] International Journal for Numerical Methods in Engineering, 2009, 77(4): 471-501.

[3] Y TANG X S, ZHENG Y R, SHI W M. Analytic solution of phreatic surface in the slope of reservoir bank [J]. Engineering Sciences, 2008, 6 (3): 2-11.

[4] MAKRODIMOPOULOS A. Remarks on some properties of conic yield restrictions in limit analysis [J]. International Journal for Numerical Methods in Biomedical Engineering, 2010, 26 (11):1449-1461.

[5] JIA Ya-jun. Soil Mechanics and Foundation engineer[M].Xi'an: Xi'an Jiaotong university Press, 2014. (in Chinese)

[6] ZHENG Yingren, WANG Yongfu, WANG Cheng. Stability analysis and exploration of failure law of jointed tunnel [J]. Chinese Journal of Underground Space and Engineering, 2011, 7(4): 649-656. 